doi: $10.2306 /$ scienceasia1513-1874.2013.39.204

\title{
Matrix versions of the classical Pólya inequality
}

\section{Limin Zou}

School of Mathematics and Statistics, Chongqing Three Gorges University, Chongqing, 404100, China

e-mail: limin-zou@163.com

Received 23 Apr 2012

Accepted 25 Jan 2013

ABSTRACT: The purpose of this paper is to give inequalities related to matrix versions of the classical Pólya inequality for scalars and discuss the relations between our results and some existing matrix inequalities.

KEYWORDS: Pólya inequality, matrix geometric mean, unitarily invariant norms

\section{INTRODUCTION}

Throughout this paper, $M_{n}$ denotes the space of $n \times$ $n$ complex matrices and $H_{n}$ denotes the set of all Hermitian matrices in $M_{n}$. For $A, B \in H_{n}$, the order relation $A \geqslant B$ means, as usual, that $A-B$ is positive semidefinite. If $A, B \in M_{n}$ are positive definite and $0 \leqslant t \leqslant 1$, the $t$-weighted geometric mean of $A$ and $B$, denoted by $A \#{ }_{t} B$, is defined as

$$
A \#_{t} B=A^{1 / 2}\left(A^{-1 / 2} B A^{-1 / 2}\right)^{t} A^{1 / 2} .
$$

When $t=\frac{1}{2}$, this is the geometric mean, denoted by $A \# B$. A norm $\|\cdot\|$ on $M_{n}$ is called unitarily invariant if

$$
\|U A V\|=\|A\|
$$

for all $A \in M_{n}$ and for all unitary matrices $U, V \in$ $M_{n}$. Throughout, $\|\cdot\|$ denotes an arbitrary unitarily invariant norm on $M_{n}$. For $A=\left[a_{i j}\right] \in M_{n}$, the Hilbert-Schmidt norm is defined by

$$
\|A\|_{2}=\left(\sum_{i, j=1}^{n}\left|a_{i j}\right|^{2}\right)^{1 / 2} .
$$

It is known that the Hilbert-Schmidt norm is unitarily invariant.

$A, B, X \in M_{n}$ such that $A$ and $B$ are positive semidefinite. Twenty years ago, Bhatia and Kittaneh $^{1,2}$ formulated some matrix versions of the arithmetic-geometric mean inequality, one of which is

$$
\left\|A^{1 / 2} X B^{1 / 2}\right\| \leqslant\left\|\frac{A X+X B}{2}\right\| .
$$

After that, a lot of interesting inequalities for matrices resulted from some classical inequalities for scalars ${ }^{3-6}$. ity:

Hiai and $\mathrm{Kosaki}^{7}$ obtained the following inequal-

$$
\begin{aligned}
\left\|A^{1 / 2} X B^{1 / 2}\right\| \leqslant\left\|\int_{0}^{1} A^{t} X B^{1-t} \mathrm{~d} t\right\| \\
\leqslant\left\|\frac{A X+X B}{2}\right\| .
\end{aligned}
$$

Meanwhile, these authors also presented a strengthening of the second inequality in (2):

$$
\begin{aligned}
\left\|\int_{0}^{1} A^{t} X B^{1-t} \mathrm{~d} t\right\| \\
\quad \leqslant \frac{1}{2}\left\|A^{1 / 2} X B^{1 / 2}+\frac{A X+X B}{2}\right\| .
\end{aligned}
$$

The inequality (2) is a refinement of the inequality (1). It is also a matrix version of the following inequality:

$$
\sqrt{a b} \leqslant \int_{0}^{1} a^{t} b^{1-t} \mathrm{~d} t \leqslant \frac{a+b}{2}, \quad a, b \geqslant 0 .
$$

Bhatia ${ }^{8}$ proved that if $\frac{1}{2} \leqslant \alpha \leqslant 1$, then

$$
\begin{aligned}
& \left\|\int_{0}^{1} A^{t} X B^{1-t} \mathrm{~d} t\right\| \\
& \quad \leqslant\left\|(1-\alpha) A^{1 / 2} X B^{1 / 2}+\alpha \frac{A X+X B}{2}\right\| .
\end{aligned}
$$

Obviously, it is a generalization of the inequality (3).

The classical Pólya inequality ${ }^{9}$ says that if $a, b \geqslant$ 0 , then

$$
\int_{0}^{1} a^{t} b^{1-t} \mathrm{~d} t \leqslant \frac{1}{3}\left(2 \sqrt{a b}+\frac{a+b}{2}\right) .
$$

In this paper, we present some matrix versions of the classical Pólya inequality and discuss the relationship between our results and some existing inequalities which are introduced above. 


\section{MAIN RESULTS}

In this section, we shall present some matrix inequalities of the Pólya type and show some related matrix inequalities.

Theorem 1 Let $A, B \in M_{n}$ be positive definite. Then

$$
\int_{0}^{1} A \#_{t} B \mathrm{~d} t \leqslant \frac{1}{3}\left(2 A \# B+\frac{A+B}{2}\right) .
$$

Proof: For a positive definite matrix $T$, it follows by the spectral theorem that there exists a unitary matrix $U \in M_{n}$ such that $T=U D U^{*}$, where $D=\operatorname{diag}\left(\lambda_{1}, \cdots, \lambda_{n}\right), \lambda_{j}>0,1 \leqslant j \leqslant n$. By inequality (5), we have

$$
\int_{0}^{1} a^{t} \mathrm{~d} t \leqslant \frac{1}{3}\left(2 \sqrt{a}+\frac{a+1}{2}\right) .
$$

Hence

$$
\int_{0}^{1} D^{t} \mathrm{~d} t \leqslant \frac{1}{3}\left(2 D^{1 / 2}+\frac{D+I}{2}\right) .
$$

Premultiplying the above inequality by $U$ and postmultiplying by $U^{*}$ gives

$$
\int_{0}^{1} T^{t} \mathrm{~d} t \leqslant \frac{1}{3}\left(2 T^{1 / 2}+\frac{T+I}{2}\right) .
$$

Putting $T=A^{-1 / 2} B A^{-1 / 2}$ in this last inequality, we obtain

$$
\begin{aligned}
\int_{0}^{1}\left(A^{-1 / 2} B A^{-1 / 2}\right)^{t} \mathrm{~d} t \leqslant & \frac{2}{3}\left(A^{-1 / 2} B A^{-1 / 2}\right)^{1 / 2} \\
& +\frac{A^{-1 / 2} B A^{-1 / 2}+I}{6} .
\end{aligned}
$$

Then, we have

$$
\begin{aligned}
A^{1 / 2} & \left(\int_{0}^{1}\left(A^{-1 / 2} B A^{-1 / 2}\right)^{t} \mathrm{~d} t\right) A^{1 / 2} \\
& \leqslant \frac{2}{3} A^{1 / 2}\left(A^{-1 / 2} B A^{-1 / 2}\right)^{1 / 2} A^{1 / 2}+\frac{A+B}{6} .
\end{aligned}
$$

That is,

$$
\int_{0}^{1} A \#_{t} B \mathrm{~d} t \leqslant \frac{1}{3}\left(2 A \# B+\frac{A+B}{2}\right) .
$$

This completes the proof.

Corollary 1 Let $A, B \in M_{n}$ be positive definite. If $\frac{1}{3} \leqslant \alpha \leqslant 1$, then

$$
\left\|\int_{0}^{1} A \#_{t} B \mathrm{~d} t\right\| \leqslant\left\|(1-\alpha) A \# B+\alpha \frac{A+B}{2}\right\| .
$$

This inequality is related to the inequality (4).

Remark 1 By the same method used in the proof of the inequality (6) and the following inequality

$$
\int_{0}^{1} a^{1-t} b^{t} \mathrm{~d} t=\int_{0}^{1} a^{t} b^{1-t} \mathrm{~d} t \leqslant \frac{1}{3}\left(2 \sqrt{a b}+\frac{a+b}{2}\right),
$$

for positive definite matrices $A, B \in M_{n}$, we have

$$
\int_{0}^{1} A \#_{1-t} B \mathrm{~d} t \leqslant \frac{1}{3}\left(2 A \# B+\frac{A+B}{2}\right) .
$$

It follows from (6) and (7) that

$$
\begin{aligned}
\int_{0}^{1}\left(\frac{A \#{ }_{t} B+A \#_{1-t} B}{2}\right) \mathrm{d} t & \\
& \leqslant \frac{1}{3}\left(2 A \# B+\frac{A+B}{2}\right),
\end{aligned}
$$

which further implies

$$
\begin{aligned}
\left\|\int_{0}^{1}\left(\frac{A \#_{t} B+A \#_{1-t} B}{2}\right) \mathrm{d} t\right\| \\
\leqslant \frac{1}{3}\left\|2 A \# B+\frac{A+B}{2}\right\| .
\end{aligned}
$$

This inequality means that if $\frac{1}{3} \leqslant \alpha \leqslant 1$, then

$$
\begin{aligned}
& \left\|\int_{0}^{1}\left(\frac{A \#{ }_{t} B+A \#_{1-t} B}{2}\right) \mathrm{d} t\right\| \\
& \leqslant\left\|(1-\alpha) A \# B+\alpha \frac{A+B}{2}\right\| .
\end{aligned}
$$

Remark 2 By the same method used in the proof of the inequality (6) and the following inequality

$$
a^{v} b^{1-v}+a^{1-v} b^{v} \leqslant 2 \int_{0}^{1} a^{t} b^{1-t} \mathrm{~d} t
$$

where

$$
\frac{1}{2}-\frac{1}{2 \sqrt{3}} \leqslant v \leqslant \frac{1}{2}+\frac{1}{2 \sqrt{3}},
$$

for positive definite matrices $A, B \in M_{n}$, we have

$$
A \#_{v} B+A \#_{1-v} B \leqslant 2 \int_{0}^{1} A \#_{t} B \mathrm{~d} t .
$$

This inequality implies

$$
\left\|A \#_{v} B+A \#_{1-v} B\right\| \leqslant 2\left\|\int_{0}^{1} A \#_{t} B \mathrm{~d} t\right\|,
$$

where

$$
\frac{1}{2}-\frac{1}{2 \sqrt{3}} \leqslant v \leqslant \frac{1}{2}+\frac{1}{2 \sqrt{3}} .
$$


Theorem 2 If $A, B, X \in M_{n}$ such that $A$ and $B$ are positive semidefinite, then

$$
\begin{aligned}
& \left\|\int_{0}^{1} A^{t} X B^{1-t} \mathrm{~d} t\right\|_{2} \\
& \quad \leqslant \frac{1}{3}\left\|2 A^{1 / 2} X B^{1 / 2}+\frac{A X+X B}{2}\right\|_{2} .
\end{aligned}
$$

Proof: Since $A$ and $B$ are positive semidefinite, it follows by the spectral theorem that there exist unitary matrices $U, V \in M_{n}$ such that $A=U \Lambda_{1} U^{*}$ and $B=V \Lambda_{2} V^{*}$, where $\Lambda_{1}=\operatorname{diag}\left(\lambda_{1}, \cdots, \lambda_{n}\right), \Lambda_{2}=$ $\operatorname{diag}\left(\mu_{1}, \cdots, \mu_{n}\right)$, with $\lambda_{i}, \mu_{i} \geqslant 0, i=1, \ldots, n$. Let $Y=U^{*} X V=\left[y_{i j}\right]$. Then

$$
\begin{aligned}
\int_{0}^{1} A^{t} X B^{1-t} \mathrm{~d} t & =\int_{0}^{1}\left(U \Lambda_{1} U^{*}\right)^{t} X\left(V \Lambda_{2} V^{*}\right)^{1-t} \mathrm{~d} t \\
& =\int_{0}^{1}\left(U \Lambda_{1}^{t} U^{*}\right) X\left(V \Lambda_{2}^{1-t} V^{*}\right) \mathrm{d} t \\
& =\int_{0}^{1} U \Lambda_{1}^{t}\left(U^{*} X V\right) \Lambda_{2}^{1-t} V^{*} \mathrm{~d} t \\
& =\int_{0}^{1} U \Lambda_{1}^{t} Y \Lambda_{2}^{1-t} V^{*} \mathrm{~d} t \\
& =U\left(\int_{0}^{1} \Lambda_{1}^{t} Y \Lambda_{2}^{1-t} \mathrm{~d} t\right) V^{*}
\end{aligned}
$$

Therefore

$$
\begin{aligned}
\left\|\int_{0}^{1} A^{t} X B^{1-t} \mathrm{~d} t\right\|_{2}^{2} & =\left\|\int_{0}^{1} \Lambda_{1}^{t} Y \Lambda_{2}^{1-t} \mathrm{~d} t\right\|_{2}^{2} \\
& =\sum_{i, j=1}^{n}\left(\int_{0}^{1} \lambda_{i}^{t} \mu_{j}^{1-t} \mathrm{~d} t\right)^{2}\left|y_{i j}\right|^{2} .
\end{aligned}
$$

Similarly, we have

$$
\begin{aligned}
\left\|2 A^{1 / 2} X B^{1 / 2}+\frac{A X+X B}{2}\right\|_{2}^{2} \\
\quad=\left(\sum_{i, j=1}^{n}\left(2 \sqrt{\lambda_{i} \mu_{j}}+\frac{\lambda_{i}+\mu_{j}}{2}\right)^{2}\left|y_{i j}\right|^{2}\right) .
\end{aligned}
$$

By the Pólya inequality for scalars, we have

$$
\begin{aligned}
& \sum_{i, j=1}^{n}\left(\int_{0}^{1} \lambda_{i}^{t} \mu_{j}^{1-t} \mathrm{~d} t\right)^{2}\left|y_{i j}\right|^{2} \\
& \quad \leqslant \frac{1}{9}\left(\sum_{i, j=1}^{n}\left(2 \sqrt{\lambda_{i} \mu_{j}}+\frac{\lambda_{i}+\mu_{j}}{2}\right)^{2}\left|y_{i j}\right|^{2}\right) .
\end{aligned}
$$

This completes the proof.
Remark 3 The Pólya matrix inequality (8) is sharper than the inequality (3) for the Hilbert-Schmidt norm. In fact, in a manner similar to the steps used to obtain (9), we have

$$
\begin{aligned}
l_{1}= & \frac{1}{4}\left\|A^{1 / 2} X B^{1 / 2}+\frac{A X+X B}{2}\right\|_{2}^{2} \\
& =\frac{1}{4}\left(\sum_{i, j=1}^{n}\left(\sqrt{\lambda_{i} \mu_{j}}+\frac{\lambda_{i}+\mu_{j}}{2}\right)^{2}\left|y_{i j}\right|^{2}\right)
\end{aligned}
$$

and

$$
\begin{aligned}
l_{2} & =\frac{1}{9}\left\|2 A^{1 / 2} X B^{1 / 2}+\frac{A X+X B}{2}\right\|_{2}^{2} \\
& =\frac{1}{9}\left(\sum_{i, j=1}^{n}\left(2 \sqrt{\lambda_{i} \mu_{j}}+\frac{\lambda_{i}+\mu_{j}}{2}\right)^{2}\left|y_{i j}\right|^{2}\right) .
\end{aligned}
$$

Hence

$$
\begin{aligned}
& l_{1}-l_{2}= \\
& \sum_{i, j=1}^{n}\left(\begin{array}{c}
\left(\frac{1}{2} \sqrt{\lambda_{i} \mu_{j}}+\frac{1}{2}\left(\frac{\lambda_{i}+\mu_{j}}{2}\right)\right)^{2} \\
-\left(\frac{2}{3} \sqrt{\lambda_{i} \mu_{j}}+\frac{1}{3}\left(\frac{\lambda_{i}+\mu_{j}}{2}\right)\right)^{2}
\end{array}\right)\left|y_{i j}\right|^{2} \\
& =\frac{1}{6} \sum_{i, j=1}^{n}\left(\begin{array}{l}
\left(\frac{\lambda_{i}+\mu_{j}}{2}-\sqrt{\lambda_{i} \mu_{j}}\right) \\
\frac{7}{6} \sqrt{\lambda_{i} \mu_{j}}+\frac{5}{6}\left(\frac{\lambda_{i}+\mu_{j}}{2}\right)
\end{array}\right)\left|y_{i j}\right|^{2}
\end{aligned}
$$

This inequality implies

$$
\begin{aligned}
& \frac{1}{3} \| 2 A^{1 / 2} X B^{1 / 2}+\frac{A X+X B}{2} \|_{2} \\
& \leqslant \frac{1}{2}\left\|A^{1 / 2} X B^{1 / 2}+\frac{A X+X B}{2}\right\|_{2} .
\end{aligned}
$$

Remark 4 An inequality weaker than (8) is

$$
\begin{aligned}
& \left\|\int_{0}^{1} A^{t} X B^{1-t} \mathrm{~d} t\right\|_{2} \\
& \quad \leqslant \frac{2}{3}\left\|A^{1 / 2} X B^{1 / 2}\right\|_{2}+\left\|\frac{A X+X B}{6}\right\|_{2} .
\end{aligned}
$$

This is also a matrix version of the classical Pólya inequality and it is a refinement of the second inequality in (2) for the Hilbert-Schmidt norm. In view of the inequalities (3) and (10), we want to know the relationship between them for the Hilbert-Schmidt norm. It should be noticed that neither (3) nor (10) is uniformly better than the other for the Hilbert-Schmidt norm. We give two examples: 
Example 1 Let

$$
\begin{aligned}
A=\left(\begin{array}{ll}
3.4029 & 3.6093 \\
3.6093 & 3.8283
\end{array}\right), X & =\left(\begin{array}{ll}
2.5870 & 0.9160 \\
1.8520 & 4.6356
\end{array}\right), \\
B & =\left(\begin{array}{ll}
2.5877 & 3.5370 \\
3.5370 & 6.6191
\end{array}\right) .
\end{aligned}
$$

We have

$$
\frac{1}{2}\left\|A^{1 / 2} X B^{1 / 2}+\frac{A X+X B}{2}\right\|_{2}=40.1635
$$

and

$$
\frac{2}{3}\left\|A^{1 / 2} X B^{1 / 2}\right\|_{2}+\left\|\frac{A X+X B}{6}\right\|_{2}=40.1940 .
$$

Example 2 Let

$$
\begin{array}{r}
A=\left(\begin{array}{ll}
4.7484 & 3.6017 \\
3.6017 & 4.0032
\end{array}\right), X=\left(\begin{array}{ll}
0.1751 & 2.7032 \\
0.3509 & 2.4482
\end{array}\right), \\
B=\left(\begin{array}{cc}
23.2871 & 11.8153 \\
11.8153 & 6.0231
\end{array}\right) .
\end{array}
$$

We have

$$
\frac{1}{2}\left\|A^{1 / 2} X B^{1 / 2}+\frac{A X+X B}{2}\right\|_{2}=34.2681
$$

and

$$
\frac{2}{3}\left\|A^{1 / 2} X B^{1 / 2}\right\|_{2}+\left\|\frac{A X+X B}{6}\right\|_{2}=331671 .
$$

Corollary 2 Let $A, B, X \in M_{n}$ such that $A$ and $B$ are positive semidefinite. If $\frac{1}{3} \leqslant \alpha \leqslant 1$, then

$$
\begin{aligned}
& \left\|\int_{0}^{1} A^{t} X B^{1-t} \mathrm{~d} t\right\|_{2} \leqslant \\
& \left\|(1-\alpha) A^{1 / 2} X B^{1 / 2}+\alpha \frac{A X+X B}{2}\right\|_{2} .
\end{aligned}
$$

Proof: Let

$$
f(x)=\left\|(1-x) A^{1 / 2} X B^{1 / 2}+x \frac{A X+X B}{2}\right\|_{2}^{2}
$$

where $0 \leqslant x \leqslant 1$. Next, we prove that if $0 \leqslant x_{1} \leqslant$ $x_{2} \leqslant 1$, then $f\left(x_{1}\right) \leqslant f\left(x_{2}\right)$. In a manner similar to the steps used to obtain (9), we have

$$
\begin{gathered}
f\left(x_{1}\right)=\left\|\left(1-x_{1}\right) A^{1 / 2} X B^{1 / 2}+x_{1} \frac{A X+X B}{2}\right\|_{2}^{2} \\
=\sum_{i, j=1}^{n}\left(\left(1-x_{1}\right) \sqrt{\lambda_{i} \mu_{j}}+x_{1} \frac{\lambda_{i}+\mu_{j}}{2}\right)^{2}\left|y_{i j}\right|^{2}
\end{gathered}
$$

and

$$
\begin{array}{r}
f\left(x_{2}\right)=\left\|\left(1-x_{2}\right) A^{1 / 2} X B^{1 / 2}+x_{2} \frac{A X+X B}{2}\right\|_{2}^{2} \\
=\sum_{i, j=1}^{n}\left(\left(1-x_{2}\right) \sqrt{\lambda_{i} \mu_{j}}+x_{2} \frac{\lambda_{i}+\mu_{j}}{2}\right)^{2}\left|y_{i j}\right|^{2} .
\end{array}
$$

By a small calculation, we have $f\left(x_{2}\right)-f\left(x_{1}\right)$ equal to

$$
\sum_{i, j=1}^{n}\left(\begin{array}{c}
\left(\begin{array}{c}
2 \sqrt{\lambda_{i} \mu_{j}}+\left(x_{2}+x_{1}\right) \\
\left(\frac{\lambda_{i}+\mu_{j}}{2}-\sqrt{\lambda_{i} \mu_{j}}\right)
\end{array}\right) \\
\times\left(x_{2}-x_{1}\right)\left(\frac{\lambda_{i}+\mu_{j}}{2}-\sqrt{\lambda_{i} \mu_{j}}\right)
\end{array}\right)\left|y_{i j}\right|^{2}
$$

which is $\geqslant 0$. The inequality (8) then shows that (11) is true for $\frac{1}{3} \leqslant \alpha \leqslant 1$. This completes the proof.

Obviously, the inequality (11) is a generalization of the inequality (4) for the Hilbert-Schmidt norm.

Acknowledgements: The author wishes to express his heartfelt thanks to the referees and editors for their detailed and helpful suggestions for revising the manuscript. This study was supported by Scientific Research Project of Southeast University.

\section{REFERENCES}

1. Bhatia R, Kittaneh F (1990) On the singular values of a product of operators. SIAM J Matrix Anal Appl 11, 272-7.

2. Bhatia R, Davis C (1993) More matrix forms of the arithmetic-geometric mean inequality. SIAM J Matrix Anal Appl 14, 132-6.

3. Ando T (1995) Matrix Young inequalities. Oper Theory Adv Appl 75, 33-8.

4. Kosaki H (1998) Arithmetic-Geometric mean and related inequalities for operators. J Funct Anal 156, 429-51.

5. Bourin JC (2006) Matrix versions of some classical inequalities. Lin Algebra Appl 416, 890-907.

6. Chansangiam P (2010) Bohr inequalities in $C^{*}$ - algebras. Sci Asia 36, 326-32.

7. Hiai F, Kosaki H (1999) Means for matrices and comparison of their norms. Indiana Univ Math J 48, 899-936.

8. Bhatia R (2006) Interpolating the arithmetic-geometric mean inequality and its operator version. Lin Algebra Appl 413, 355-63.

9. Niculescu CP, Persson LE (2006) Convex Functions and Their Applications: A Contemporary Approach, CMS Books in Mathematics, Vol. 23, Springer-Verlag, New York. 